\title{
Existence of positive solution for a fractional order nonlinear differential system involving a changing sign perturbation
}

\author{
Jianxin $\mathrm{He}^{\mathrm{a}, \mathrm{b}, *}$, Xinguang Zhang ${ }^{\mathrm{c}, \mathrm{d}, *}$, Yonghong $\mathrm{Wu}^{\mathrm{d}}$ \\ ${ }^{a}$ School of Science, Nanjing University of Science and Technology, Nanjing, 210094, P. R. China. \\ ${ }^{b}$ School of Science, Nanjing University of Posts and Telecommunications, Nanjing, 210023, P. R. China. \\ 'School of Mathematical and Informational Sciences, Yantai University, Yantai 264005, Shandong, China. \\ ${ }^{d}$ Department of Mathematics and Statistics, Curtin University of Technology, Perth, WA 6845, Australia.
}

Communicated by Y. Yao

\begin{abstract}
In this paper, we study a class of singular fractional order differential system with a changing-sign perturbation which arises from fluid dynamics, biological models, electrical networks with uncertain physical parameters and parametrical variations in time. Under suitable growth condition, the singular changingsign system is transformed to an approximately singular fractional order differential system with positive nonlinear term, then the existence of positive solution is established by using the known fixed point theorem. (C)2016 All rights reserved.
\end{abstract}

Keywords: Singular phenomena, changing-sign perturbation, positive solution, Riemann-Stieltjes integral conditions.

2010 MSC: 34B15, 34B18.

\section{Introduction}

The study of many problems in fluid dynamics, biological models, electrical networks, chemical kinetics often leads to mathematical models in the form of nonlinear fractional order differential equations. Generally,

\footnotetext{
${ }^{*}$ Corresponding author

Email addresses: hjx_cheng@163.com (Jianxin He), zxg123242@163.com (Xinguang Zhang), y.wu@curtin.edu.au (Yonghong Wu)
} 
some perturbations and uncertainties usually exist in these real world differential models due to some uncertain physical parameters and parametrical variations in time. These perturbations and uncertainties can be introduced in the underlying mathematical model [3, 5, 7, 9, 10, 15, 16, 18, .

In this paper, we consider a class of singular fractional order differential system with a changing-sign perturbation

$$
\left\{\begin{array}{l}
-D_{0+}^{\alpha} u(t)=f(t, v(t))+q(t), \quad-D_{0+}^{\beta} v(t)=g(t, u(t)), 0<t<1, \\
u(0)=0, u(1)=\int_{0}^{1} u(s) d A(s), v(0)=0, v(1)=\int_{0}^{1} v(s) d B(s),
\end{array}\right.
$$

where $1<\alpha, \beta \leq 2, D_{0+}^{\alpha}$ and $D_{0+}^{\beta}$ are the standard Riemann-Liouville derivatives, $A$ and $B$ are functions of bounded variation, $\int_{0}^{1} u(s) d A(s)$ and $\int_{0}^{1} v(s) d B(s)$ denote the Riemann-Stieltjes integrals of $u, v$ with respect to $A$ and $B, f, g \in C((0,1) \times[0,+\infty),[0,+\infty)), f$ and $g$ may be singular at $t=0$ and $t=1$, $q:(0,1) \rightarrow(-\infty,+\infty)$ is Lebesgue integrable and $q$ can have infinitely many singularities in $[0,1]$.

In recent years, a handful of papers have appeared to study differential equation with some changingsign perturbation, but most of them treated with the changing-sign perturbation problems with the form $f(t, u)+M \geq 0$ for some $M>0$ [1, 11], especially, containing fractional derivatives, for detail, see [8, 14, 17, 19, 20, 21, 22, 23, 24. In this paper, we handle the singular fractional order differential system with the form $f(t, u)+q(t) \rightarrow-\infty$ at some singular point. By finding the unique solution of the linear nonlocal boundary value problem, we transform the changing-sign singular fractional order differential system to an approximately positive differential system, and then the existence of positive solution is established by using the known fixed point theorem. Here we also point out the boundary condition of system (1.1) is nonlocal which involves the Riemann-Stieltjes integral, i.e. it can cover the multi-point BCs and also integral BCs in a single framework.

\section{Basic Definitions and Preliminaries}

In this paper, we restrict our attention to the Riemann-Liouville fractional derivatives and some properties of the Riemann-Liouville fractional integral and derivative operators, see $[\underline{6}, 12,13$.

Lemma $2.1([2])$. Given $h(t) \in L^{1}(0,1)$, the problem

$$
\left\{\begin{array}{l}
-D_{0+}^{\alpha} u(t)=h(t), \quad 0<t<1, \\
u(0)=0, \quad u(1)=0
\end{array}\right.
$$

has the unique solution

$$
u(t)=\int_{0}^{1} G_{A}(t, s) h(s) d s,
$$

where $G_{A}(t, s)$ is the Green function of (2.1) and is given by

$$
G_{A}(t, s)=\frac{1}{\Gamma(\alpha)} \begin{cases}{[t(1-s)]^{\alpha-1},} & 0 \leq t \leq s \leq 1 \\ {[t(1-s)]^{\alpha-1}-(t-s)^{\alpha-1},} & 0 \leq s \leq t \leq 1\end{cases}
$$

Lemma 2.2 ([14]). If $h(t) \in L^{1}(0,1)$, the Green function of the following problem

$$
\left\{\begin{array}{l}
-D_{0+}^{\alpha} u(t)=h(t), 0<t<1 \\
u(0)=0, u(1)=\int_{0}^{1} u(s) d A(s)
\end{array}\right.
$$

is given by

$$
H_{A}(t, s)=\frac{t^{\alpha-1}}{1-\mathcal{A}} \mathcal{G}_{A}(s)+G_{A}(t, s), \quad \mathcal{A}=\int_{0}^{1} t^{\alpha-1} d A(t)
$$


where

$$
\mathcal{G}_{A}(s)=\int_{0}^{1} G_{A}(t, s) d A(t) .
$$

Lemma 2.3 ([14]). Let $0 \leq \mathcal{A}<1, \mathcal{G}_{A} \geq 0$ for $s \in[0,1]$, then the Green function defined by (2.5) satisfies:

(1) $H_{A}(t, s)>0$ for all $(t, s) \in(0,1) \times(0,1)$,

(2) There exist two constants $c_{A}, d_{A}$ such that

$$
c_{A} t^{\alpha-1} \mathcal{G}_{A}(s) \leq H_{A}(t, s) \leq d_{A} t^{\alpha-1} \leq d_{A}, \quad t, s \in[0,1] .
$$

Here we also address that for the equation

$$
\left\{\begin{array}{l}
-D_{0+}^{\beta} v(t)=h(t), 0<t<1 \\
v(0)=0, v(1)=\int_{0}^{1} v(s) d B(s)
\end{array}\right.
$$

we can get results similar to (2.3) and (2.5) as well as (2.6), and the results will be denoted by subscript $B$.

For clarity in presentation, we also list below some assumptions to be used later in the paper.

(H1) $A, B$ are functions of bounded variation such that $\mathcal{G}_{A}(s) \geq 0, \mathcal{G}_{B}(s) \geq 0$ for $s \in[0,1]$ and $0 \leq \mathcal{A}, \mathcal{B}<1$;

(H2) $f, g:(0,1) \times[0,+\infty) \rightarrow[0,+\infty)$ are continuous, and are nondecreasing on second variable, and there exist two continuous functions $p:(0,1) \rightarrow[0,+\infty)$ and $h:[0,+\infty) \rightarrow[0,+\infty)$ such that

$$
f(t, u) \leq p(t) h(u) .
$$

(H3) There exists a constant $\lambda>0$ such that for any $(t, v) \in(0,1) \times[0,+\infty)$

$$
g(t, c v) \geq c^{\lambda} g(t, v), \forall 0<c \leq 1,
$$

with $0<\int_{0}^{1} g(t, 1) d t<+\infty$.

(H4) $q:(0,1) \rightarrow(-\infty,+\infty)$ is Lebesgue integrable such that $0<\int_{0}^{1} q_{-}(t) d t<c_{A}^{-1} d_{A}^{2}$ and

$$
\int_{0}^{1}\left[p(s)+q_{+}(s)\right] d s \leq d_{A}^{-1}\left(\max _{0 \leq \tau \leq M} h(\tau)+1\right)^{-1},
$$

where

$$
q_{+}(t)=\max \{q(t), 0\}, q_{-}(t)=\min \{q(t), 0\}, M=d_{B} \int_{0}^{1} g(\tau, 1) d \tau
$$

$$
\lim _{u \rightarrow+\infty} \frac{f(t, u)}{u}=+\infty, \lim _{u \rightarrow+\infty} \frac{g(t, u)}{u}=+\infty
$$

for $t$ uniformly on any close subinterval of $(0,1)$.

Remark 2.4. Let $\Omega \subset(0,1)$ be a zero measure set and $q:(0,1) \backslash \Omega \rightarrow(-\infty,+\infty)$ be continuous and integrable, then $q$ can have infinitely many singularities.

Remark 2.5. If $q$ satisfies $(\mathbf{H} 4)$ and $\lim _{t \rightarrow t_{0}} q(t)=-\infty$ for some point $t_{0} \in(0,1)$, then the system can be changing-sign.

Remark 2.6. For any $(t, v) \in(0,1) \times[0,+\infty)$ and $\widetilde{c} \geq 1$, by (H3), it is easy to get

$$
g(t, \widetilde{c} v) \leq \widetilde{c}^{\lambda} g(t, v) .
$$


Throughout this paper, we will work in the space $E=C[0,1]$, which is a Banach space if it is endowed with the norm $\|u\|=\max _{t \in[0,1]}|u(t)|$ for any $u \in E$. Let

$$
P=\left\{u \in C[0,1]: u(t) \geq \frac{c_{A}}{d_{A}} t^{\alpha-1}\|u\|, t \in[0,1]\right\} .
$$

Obviously $P$ is a normal cone in the Banach space $E$. For $u \in E$, let us define a function $[\cdot]^{*}$ by

$$
[u(t)]^{*}= \begin{cases}u(t), & u(t) \geq 0 \\ 0, & u(t)<0\end{cases}
$$

By Lemma 2.3, the following equation

$$
\left\{\begin{array}{l}
-D_{0+}^{\alpha} u(t)=q_{-}(t), 0<t<1 \\
u(0)=0, u(1)=\int_{0}^{1} u(s) d A(s)
\end{array}\right.
$$

has unique solution which satisfies

$$
w(t)=\int_{0}^{1} H_{A}(t, s) q_{-}(s) d s \leq d_{A} \int_{0}^{1} q_{-}(s) d s .
$$

In what follows, we consider the following approximately singular fractional order differential system

$$
\left\{\begin{array}{l}
-D_{0+}^{\alpha} u(t)=f(t, v(t))+q_{+}(t), \quad-D_{0+}^{\beta} v(t)=g\left(t,[u(t)-w(t)]^{*}\right), 0<t<1, \\
u(0)=0, u(1)=\int_{0}^{1} u(s) d A(s), v(0)=0, v(1)=\int_{0}^{1} v(s) d B(s)
\end{array}\right.
$$

Clearly, by Lemma 2.3, we know that the system (2.11) is equivalent to the following integral system

$$
\left\{\begin{array}{l}
u(t)=\int_{0}^{1} H_{A}(t, s)\left[f(s, v(s))+q_{+}(s)\right] d s \\
v(t)=\int_{0}^{1} H_{B}(t, s) g\left(s,[u(s)-w(s)]^{*}\right) d s .
\end{array}\right.
$$

Obviously, the above nonlinear integral system 2.12 can be transformed to the following equivalent nonlinear integral equation

$$
u(t)=\int_{0}^{1} H_{A}(t, s)\left[f\left(s, \int_{0}^{1} H_{B}(s, \tau) g\left(\tau,[u(\tau)-w(\tau)]^{*}\right) d \tau\right)+q_{+}(s)\right] d s .
$$

Now let us define a nonlinear integral operator $T: E \rightarrow E$ by

$$
(T u)(t)=\int_{0}^{1} H_{A}(t, s)\left[f\left(s, \int_{0}^{1} H_{B}(s, \tau) g\left(\tau,[u(\tau)-w(\tau)]^{*}\right) d \tau\right)+q_{+}(s)\right] d s, t \in[0,1],
$$

we have that if $u(t)$ is a fixed point of $T$ in $E$, then system 2.11) has one solution $(u, v)$ with

$$
\left\{\begin{aligned}
u & =u(t) \\
v & =\int_{0}^{1} H_{B}(t, s) g\left(s,[u(s)-w(s)]^{*}\right) d s
\end{aligned}\right.
$$

Lemma 2.7. If $(u, v)$ with $u(t) \geq w(t)$ for any $t \in[0,1]$ is a positive solution of approximately singular fractional order differential system (2.11), then $(u-w, v)$ is a positive solution of singular fractional order differential system with a negative perturbation (1.1), where 


$$
v(t)=\int_{0}^{1} H_{B}(t, s) g(s, u(s)-w(s)) d s .
$$

Proof. In fact, if $(u, v)$ is a positive solution system (2.11) and satisfies $u(t) \geq w(t)$ for any $t \in[0,1]$, then, by (2.11) and the definition of $[u(t)]^{*}$, we have

$$
\left\{\begin{array}{l}
-D_{0+}^{\alpha} u(t)=f(t, v(t))+q_{+}(t), \quad-D_{0+}^{\beta} v(t)=g(t, u(t)-w(t)), \\
u(0)=0, u(1)=\int_{0}^{1} u(s) d A(s), v(0)=0, v(1)=\int_{0}^{1} v(s) d B(s) .
\end{array}\right.
$$

Let $u_{1}=u-w$, then we have

$$
\begin{aligned}
& D_{0+}^{\alpha} u_{1}(t)=D_{0+}^{\alpha} u(t)-D_{0+}^{\alpha} w(t)=D_{0+}^{\alpha} u(t)+q_{-}(t), \\
& u_{1}(0)=0, u_{1}(1)=\int_{0}^{1} u_{1}(s) d A(s)
\end{aligned}
$$

which implies that

$$
\left\{\begin{array}{l}
-D_{0+}^{\alpha} u_{1}(t)+q_{-}(t)=f(t, v(t))+q_{+}(t), \quad-D_{0+}^{\beta} v(t)=g\left(t, u_{1}(t)\right), \\
u_{1}(0)=0, u_{1}(1)=\int_{0}^{1} u_{1}(s) d A(s), v(0)=0, v(1)=\int_{0}^{1} v(s) d B(s) .
\end{array}\right.
$$

Notice that $q(t)=q_{+}(t)-q_{-}(t)$, we have $\left(u_{1}, v\right)=(u-w, v)$ is a positive solution of singular fractional order differential system with a negative perturbation (1.1).

Lemma 2.8. $T: P \rightarrow P$ is a completely continuous operator.

Proof. For any fixed $u \in P$, we can find a constant $L_{1}>0$ such that $\|u\| \leq L_{1}$. Take $\hat{L}=\max \left\{L_{1}, 1\right\}$, then

$$
0 \leq[u(s)-w(s)]^{*} \leq u(s) \leq\|u\| \leq L_{1} \leq \hat{L} .
$$

It follows from (2.17) and (H2)-(H3) that

$$
g\left(\tau,[u(\tau)-w(\tau)]^{*}\right) \leq g(\tau, \hat{L}) \leq \hat{L}^{\lambda} g(\tau, 1) .
$$

And then

$$
\int_{0}^{1} H_{B}(s, \tau) g\left(\tau,[u(\tau)-w(\tau)]^{*}\right) d \tau \leq d_{B} \int_{0}^{1} \hat{L}^{\lambda} g(\tau, 1) d \tau=d_{B} \hat{L}^{\lambda} \int_{0}^{1} g(\tau, 1) d \tau:=\hat{M}
$$

Consequently, for any $t \in[0,1]$, we have

$$
\begin{aligned}
(T u)(t) & =\int_{0}^{1} H_{A}(t, s)\left[f\left(s, \int_{0}^{1} H_{B}(s, \tau) g\left(\tau,[u(\tau)-w(\tau)]^{*}\right) d \tau\right)+q_{+}(s)\right] d s \\
& \leq d_{A} \int_{0}^{1}\left[p(s) h\left(\int_{0}^{1} H_{B}(s, \tau) g\left(\tau,[u(\tau)-w(\tau)]^{*}\right) d \tau\right)+q_{+}(s)\right] d s \\
& \leq d_{A} \int_{0}^{1}\left[p(s) h\left(d_{B} \hat{L}^{\lambda} \int_{0}^{1} g(\tau, 1) d \tau\right)+q_{+}(s)\right] d s \\
& \leq d_{A}\left(\max _{0 \leq \tau \leq \hat{M}} h(\tau)+1\right) \int_{0}^{1}\left[p(s)+q_{+}(s)\right] d s<+\infty
\end{aligned}
$$

which implies that the operator $T: P \rightarrow E$ is bounded. 
Next for any $u \in P$, by Lemma 2.3 , we have

$$
\begin{aligned}
\|T u\| & =\max _{0 \leq t \leq 1}\left\{\int_{0}^{1} H_{A}(t, s)\left[f\left(s, \int_{0}^{1} H_{B}(s, \tau) g\left(\tau,[u(\tau)-w(\tau)]^{*}\right) d \tau\right)+q_{+}(s)\right] d s\right\} \\
& \leq d_{A} \int_{0}^{1}\left[f\left(s, \int_{0}^{1} H_{B}(s, \tau) g\left(\tau,[u(\tau)-w(\tau)]^{*}\right) d \tau\right)+q_{+}(s)\right] d s .
\end{aligned}
$$

On the other hand, by Lemma 2.3 , we also have

$$
(T u)(t) \geq c_{A} t^{\alpha-1} \int_{0}^{1}\left[f\left(s, \int_{0}^{1} H_{B}(s, \tau) g\left(\tau,[u(\tau)-w(\tau)]^{*}\right) d \tau\right)+q_{+}(s)\right] d s .
$$

So, 2.18) and 2.19) yield

$$
(T u)(t) \geq \frac{c_{A}}{d_{A}}\|T u\| t^{\alpha-1}, t \in[0,1],
$$

which yields that $T(P) \subset P$.

At the end, using standard arguments, according to the Ascoli-Arzela Theorem, one can show that $T: P \rightarrow P$ is a completely continuous operator.

\section{Main results}

Theorem 3.1. Suppose (H1) - (H5) hold. Then the singular fractional order differential system with a changing-sign perturbation (1.1) has at least one positive solution.

Proof. Take $\Omega_{1}=\{u \in P:\|u\| \leq 1\}$, then for any $u \in \partial \Omega_{1}$, we have

$$
0 \leq[u(s)-w(s)]^{*} \leq u(s) \leq\|u\| \leq 1 .
$$

Thus for any $u \in \partial \Omega_{1}$, it follows from (3.1) and (H2)-(H3) that

$$
\begin{aligned}
\|T u\| & =\max _{0 \leq t \leq 1}\left\{\int_{0}^{1} H_{A}(t, s)\left[f\left(s, \int_{0}^{1} H_{B}(s, \tau) g\left(\tau,[u(\tau)-w(\tau)]^{*}\right) d \tau\right)+q_{+}(s)\right] d s\right\} \\
& \leq d_{A} \int_{0}^{1}\left[p(s) h\left(\int_{0}^{1} H_{B}(s, \tau) g\left(\tau,[u(\tau)-w(\tau)]^{*}\right) d \tau\right)+q_{+}(s)\right] d s \\
& \leq d_{A} \int_{0}^{1}\left[p(s) h\left(d_{B} \int_{0}^{1} g(\tau, 1) d \tau\right)+q_{+}(s)\right] d s \\
& \leq d_{A}\left(\max _{0 \leq \tau \leq M} h(\tau)+1\right) \int_{0}^{1}\left[p(s)+q_{+}(s)\right] d s \\
& \leq 1=\|u\|,
\end{aligned}
$$

where $M=d_{B} \int_{0}^{1} g(\tau, 1) d \tau$. Therefore,

$$
\|T u\| \leq\|u\|, \quad u \in \partial \Omega_{1} .
$$

On the other hand, choose constants $a, b$ and $L$ such that

$$
[a, b] \subset(0,1), \frac{c_{A}}{2 d_{A}} a^{\alpha-1} L \int_{a}^{b} \frac{\mathcal{G}_{A}(s)}{1-\mathcal{A}} d s>1 .
$$

By (H5), there exists a constant enough large

$$
K^{*}>\max \left\{2 c_{A} \int_{0}^{1} q_{-}(s) d s, 1\right\}
$$


such that

$$
f(t, x) \geq L x, t \in[a, b], x \geq K^{*} .
$$

Furthermore, from (H5), there exists a constant $K>K^{*}$ such that

$$
g(t, y) \geq\left(a^{\alpha-1} c_{A} \int_{a}^{b} \mathcal{G}_{A}(\tau) d \tau\right)^{-1} y, t \in[a, b], y \geq K .
$$

Now take $R \geq \max \left\{\frac{2 K d_{A}}{c_{A} a^{\alpha-1}}, K\right\}$. Obviously,

$$
R \geq K>K^{*}>\max \left\{2 c_{A} \int_{0}^{1} q_{-}(s) d s, 1\right\} .
$$

Let $\Omega_{2}=\{u \in P:\|u\| \leq R\}$. Then for any $u \in \partial \Omega_{2}$ and for any $t \in[a, b]$, we have

$$
\begin{aligned}
u(t)-w(t) & \geq u(t)-d_{A} t^{\alpha-1} \int_{0}^{1} q_{-}(s) d s \geq u(t)-c_{A} \int_{0}^{1} q_{-}(s) d s \frac{u(t)}{\|u\|} \\
& =u(t)-c_{A} \int_{0}^{1} q_{-}(s) d s \frac{u(t)}{R} \geq \frac{1}{2} u(t) \\
& \geq \frac{c_{A}}{2 d_{A}} t^{\alpha-1} R \geq \frac{c_{A}}{2 d_{A}} a^{\alpha-1} R \geq K>0 .
\end{aligned}
$$

And then, it follows from (3.5) that, for any $s \in[a, b]$,

$$
\begin{aligned}
\int_{a}^{b} H_{B}(s, \tau) g\left(\tau,[u(\tau)-w(\tau)]^{*}\right) d \tau & =\int_{a}^{b} H_{B}(s, \tau) g(\tau, u(\tau)-w(\tau)) d \tau \\
& \geq\left(a^{\alpha-1} c_{A} \int_{a}^{b} \mathcal{G}_{A}(\tau) d \tau\right)^{-1} \int_{a}^{b} c_{A} s^{\alpha-1} \mathcal{G}_{A}(\tau)(u(\tau)-w(\tau)) d \tau \\
& \geq \frac{c_{A}}{2 d_{A}} a^{\alpha-1} R \geq K>K^{*}>0 .
\end{aligned}
$$

So for any $u \in \partial \Omega_{2}$ and $t \in[0,1]$, by (3.4) and (3.6), we have

$$
\begin{aligned}
\|T u\| & \geq \int_{0}^{1} H_{A}(1, s)\left[f\left(s, \int_{0}^{1} H_{B}(s, \tau) g\left(\tau,[u(\tau)-w(\tau)]^{*}\right) d \tau\right)+q_{+}(s)\right] d s \\
& \geq \int_{0}^{1} \frac{\mathcal{G}_{A}(s)}{1-\mathcal{A}} f\left(s, \int_{0}^{1} H_{B}(s, \tau) g\left(\tau,[u(\tau)-w(\tau)]^{*}\right) d \tau\right) d s \\
& \geq \int_{a}^{b} \frac{\mathcal{G}_{A}(s)}{1-\mathcal{A}} f\left(s, \int_{a}^{b} H_{B}(s, \tau) g\left(\tau,[u(\tau)-w(\tau)]^{*}\right) d \tau\right) d s \\
& \geq L \int_{a}^{b} \frac{\mathcal{G}_{A}(s)}{1-\mathcal{A}} \int_{a}^{b} H_{B}(s, \tau) g\left(\tau,[u(\tau)-w(\tau)]^{*}\right) d \tau d s \\
& \geq \frac{c_{A}}{2 d_{A}} a^{\alpha-1} R L \int_{a}^{b} \frac{\mathcal{G}_{A}(s)}{1-\mathcal{A}} d s \geq R=\|u\| .
\end{aligned}
$$

Thus, we have $\|T u\| \geq\|u\|, u \in \partial \Omega_{2}$. Thus $T$ has a fixed point $u_{0}$ such that $1 \leq\left\|u_{0}\right\| \leq R$ from [4].

In what follows, we prove $u_{0}(t) \geq w(t)$ for any $t \in[0,1]$. In fact, for any $t \in[0,1]$, by (H4), we have

$$
\begin{aligned}
u_{0}(t)-w(t) & \geq u_{0}(t)-d_{A} t^{\alpha-1} \int_{0}^{1} q_{-}(s) d s \geq \frac{c_{A}}{d_{A}} t^{\alpha-1}-d_{A} t^{\alpha-1} \int_{0}^{1} q_{-}(s) d s \\
& \geq\left(\frac{c_{A}}{d_{A}}-d_{A} \int_{0}^{1} q_{-}(s) d s\right) t^{\alpha-1} \geq 0 .
\end{aligned}
$$

By Lemma 2.7 and (3.7), the singular fractional order differential system with a changing-sign perturbation (1.1) has at least one positive solution. The proof of Theorem 3.1 is completed. 


\section{An example}

To demonstrate the application of our results, we give a simple example. Firstly, we take $\alpha=\frac{3}{2}, \beta=\frac{4}{3}$, and

$$
A(t)=\left\{\begin{array}{ll}
0, & t \in\left[0, \frac{1}{3}\right), \\
\frac{1}{2}, & t \in\left[\frac{1}{3}, \frac{2}{3}\right), \\
1, & t \in\left[\frac{2}{3}, 1\right],
\end{array} \quad B(t)= \begin{cases}0, & t \in\left[0, \frac{1}{2}\right), \\
2, & t \in\left[\frac{1}{2}, \frac{3}{4}\right), \\
1, & t \in\left[\frac{3}{4}, 1\right] .\end{cases}\right.
$$

By Lemma 2.3, there exist some positive constants $c_{A}, d_{A}, c_{B}, d_{B}$ such that

$$
c_{A} t^{\frac{1}{2}} \mathcal{G}_{A}(s) \leq H_{A}(t, s) \leq d_{A} t^{\frac{1}{2}} \leq d_{A}, \quad t, s \in[0,1],
$$

and

$$
c_{B} t^{\frac{1}{3}} \mathcal{G}_{B}(s) \leq H_{B}(t, s) \leq d_{B} t^{\frac{1}{3}} \leq d_{B}, \quad t, s \in[0,1] .
$$

Consider the following singular fractional order differential system with a changing-sign perturbation

$$
\left\{\begin{array}{l}
-D_{0+}^{\frac{3}{2}} u(t)=d_{A}^{-1}\left(\frac{4}{9} d_{B}^{2}+1\right)^{-1} t v^{2}-\frac{c_{A}^{-1} d_{A}^{2}}{4 \sqrt{t}},-D_{0+}^{\frac{4}{3}} v(t)=\frac{u^{\frac{3}{2}}}{2 \sqrt[4]{t}}, \\
u(0)=0, u(1)=\int_{0}^{1} u(s) d A(s), v(0)=0, v(1)=\int_{0}^{1} v(s) d B(s) .
\end{array}\right.
$$

Analysis. Let

$$
\begin{aligned}
& f(t, x)=d_{A}^{-1}\left(\frac{4}{9} d_{B}^{2}+1\right)^{-1} t x^{2}, g(t, y)=\frac{y^{\frac{3}{2}}}{2 \sqrt[4]{t}}, \\
& p(t)=d_{A}^{-1}\left(\frac{4}{9} d_{B}^{2}+1\right)^{-1} t, h(x)=x^{2}, q(t)=-\frac{c_{A}^{-1} d_{A}^{2}}{4 \sqrt{t}},
\end{aligned}
$$

then we have

$$
f(t, x) \leq p(t) h(x), \quad \int_{0}^{1} g(t, 1) d t=\int_{0}^{1} \frac{1}{2 \sqrt[4]{t}} d t=\frac{2}{3} .
$$

Clearly, $f, g:(0,1) \times[0,+\infty) \rightarrow[0,+\infty)$ are continuous, and are nondecreasing on second variable, and for any $0<c \leq 1$,

$$
g(t, c y)=\frac{c^{\frac{3}{2}} y^{\frac{3}{2}}}{2 \sqrt[4]{t}} \geq \frac{c^{2} y^{\frac{3}{2}}}{2 \sqrt[4]{t}}=c^{2} g(t, y) .
$$

Thus (H2) and (H3) hold.

On the other hand, clearly,

$$
q_{-}(t)=\frac{c_{A}^{-1} d_{A}^{2}}{4 \sqrt{t}}, q_{+}(t)=0, M=d_{B} \int_{0}^{1} g(\tau, 1) d \tau=\frac{2}{3} d_{B},
$$

so

$$
d_{A}^{-1}\left(\max _{0 \leq \tau \leq M} h(\tau)+1\right)^{-1}=d_{A}^{-1}\left(\frac{4}{9} d_{B}^{2}+1\right)^{-1} .
$$

Thus, we have

$$
\begin{aligned}
& \int_{0}^{1} q_{-}(t) d t=\int_{0}^{1} \frac{c_{A}^{-1} d_{A}^{2}}{4 \sqrt{t}} d t=\frac{1}{2} c_{A}^{-1} d_{A}^{2}<c_{A}^{-1} d_{A}^{2} \\
& \int_{0}^{1}\left(p(s)+q_{+}(s)\right) d s=\int_{0}^{1} d_{A}^{-1}\left(\frac{4}{9} d_{B}^{2}+1\right)^{-1} s d s=\frac{1}{2} d_{A}^{-1}\left(\frac{4}{9} d_{B}^{2}+1\right)^{-1}<d_{A}^{-1}\left(\max _{0 \leq \tau \leq M} h(\tau)+1\right)^{-1} .
\end{aligned}
$$


So (H4) hold. Moreover, (H5) is also easy to be verified.

In addition, we have

$$
\begin{aligned}
& 0 \leq \mathcal{A}=\int_{0}^{1} t^{\frac{1}{2}} d A(t)=\frac{1}{2}\left(\frac{2}{3}\right)^{\frac{1}{2}}+\frac{1}{2}\left(\frac{1}{3}\right)^{\frac{1}{2}} \approx 0.6969<1, \\
& 0 \leq \mathcal{B}=\int_{0}^{1} t^{\frac{1}{3}} d B(t)=2\left(\frac{1}{2}\right)^{\frac{1}{3}}-\left(\frac{3}{4}\right)^{\frac{1}{3}} \approx 0.6788<1 .
\end{aligned}
$$

Clearly, $\mathcal{G}_{A}(s), \mathcal{G}_{B}(s) \geq 0$ for $s \in[0,1]$ also hold, which implies that (H1) is satisfied.

Hence all conditions of Theorem 3.1 are satisfied, and consequently from Theorem 3.1, the singular fractional order differential system with a changing-sign perturbation (4.3) has at least one positive solution.

\section{Acknowledgements}

The authors were supported financially by the National Natural Science Foundation of China 11371221, 11571296, 61503198, the Universities Natural Science Foundation of Jiangsu, China(NO.13KJD110003), the Natural Science Foundation of Jiangsu Province of China (BK20150827) and the Natural Science Foundation of Shandong Province of China (ZR2014AM009).

\section{References}

[1] R. Agarwal, D. O'Regan, A note on existence of nonnegative solutions to singular semi-positone problems, Nonlinear Anal., 36 (1999), 615-622.1.

[2] Z. Bai, H. Lv, Positive solutions for boundary value problem of nonlinear fractional differential equation, J. Math. Anal. Appl., 311 (2005), 495-505.2.1

[3] Y. Q. Chen, H. S. Ahn, I. Podlubny, Robust stability check of fractional order linear time invariant systems with interval uncertainties, Proc. IEEE Int. Conference Mech. Automation, 1 (2005), 210-215.1.

[4] D. Guo, V. Lakshmikantham, Nonlinear Problems in Abstract Cone, Academic Press, Inc., New York, (1988).3

[5] S. Hosseinnia, R. Ghaderi, A. Ranjbar, M. Mahmoudian, S. Momani, Sliding mode synchronization of an uncertain fractional order chaotic system, Comput. Math. Appl., 59 (2010), 1637-1643.1]

[6] A. Kilbas, H. Srivastava, J. Trujillo, Theory and Applications of Fractional Differential Equations, Elsevier B.V, Netherlands, (2006).2

[7] Y. H. Lan, Y. Zhou, LMI-based robust control of fractional-order uncertain linear systems, Comput. Math. Appl., 62 (2011), 1460-1471.1

[8] S. Li, X. Zhang, Y. Wu, L. Caccetta, Extremal solutions for p-Laplacian differential systems via iterative computation, Appl. Math. Lett., 26 (2013), 1151-1158.1

[9] Z. Liao, C. Peng, W. Li, Y. Wang, Robust stability analysis for a class of fractional order systems with uncertain parameters, J. Franklin Inst., 348 (2011), 1101-1113.1]

[10] T. Lin, T. Lee, Chaos synchronization of uncertain fractional-order chaotic systems with time delay based on adaptive fuzzy sliding mode control, IEEE Trans. Fuzzy Sys., 19 (2011), 623-635.1.

[11] R. Ma, R. Wang, L. Ren, Existence results for semipositone boundary value problems, Acta Math. Sci. Ser. B Engl. Ed., 21 (2001), 189-195.1

[12] K. Miller, B. Ross, An Introduction to the Fractional Calculus and Fractional Differential Equations, Wiley, New York, (1993).2

[13] I. Podlubny, Fractional Differential Equations, Mathematics in Science and Engineering, Academic Press, New York, (1999).2

[14] X. Zhang, Y. Han, Existence and uniqueness of positive solutions for higher order nonlocal fractional differential equations, Appl. Math. Lett., 25 (2012), 555-560.1, $2.2,2.3$

[15] X. Zhang, L. Liu, Positive solutions of superlinear semipositone singular Dirichlet boundary value problems, J. Math. Anal. Appl., 316 (2006), 525-537.11

[16] X. Zhang, L. Liu, Multiple positive solutions of a singular fractional differential equation with negatively perturbed term, Math. Compu. Modelling, 55 (2012), 1263-1274.1

[17] X. Zhang, L. Liu, B. Wiwatanapataphee, Y. Wu, The eigenvalue for a class of singular p-Laplacian fractional differential equations involving the Riemann-Stieltjes integral boundary condition, Appl. Math. Comput., 235 (2014), 412-422.1]

[18] X. Zhang, L. Liu, Y. Wu, Existence results for multiple positive solutions of nonlinear higher order perturbed fractional differential equations with derivatives, Appl. Math. Comput., 219 (2012), 1420-1433.1. 
[19] X. Zhang, L. Liu, Y. Wu, The eigenvalue problem for a singular higher fractional differential equation involving fractional derivatives, Appl. Math. Comput., 218 (2012), 8526-8536.1

[20] X. Zhang, L. Liu, Y. Wu, The uniqueness of positive solution for a singular fractional differential system involving derivatives, Commun. Nonlinear Sci. Numer. Simul., 18 (2013), 1400-1409.1

[21] X. Zhang, L. Liu, Y. Wu, The uniqueness of positive solution for a fractional order model of turbulent flow in a porous medium, Appl. Math. Lett., 37 (2014), 26-33.1

[22] X. Zhang, L. Liu, Y. Wu, Variational structure and multiple solutions for a fractional advection-dispersion equation, Comput. Math. Appl., 68 (2014), 1794-1805.1

[23] X. Zhang, L. Liu, Y. Wu, Y. Lu, The iterative solutions of nonlinear fractional differential equations, Appl. Math. Comput., 219 (2013), 4680-4691.1.

[24] X. Zhang, Y. Wu, L. Caccetta, Nonlocal fractional order differential equations with changing-sign singular perturbation, Appl. Math. Model., 39 (2015), 6543-6552.1 